\title{
THE ECOLOGY OF QUASAR ABSORPTION SYSTEMS
}

\author{
DAVID TYTLER \\ University of California San Diego \\ CASS 0111, La Jolla,CA 92093-0111, tytler@ucsd.edu
}

\section{Introduction}

Gas along the lines of sight to QSOs produces narrow absorption lines from ions of the most abundant elements: $\mathrm{H}, \mathrm{He}, \mathrm{C}, \mathrm{N}, \mathrm{O}, \mathrm{Si}, \mathrm{S}, \mathrm{Mg}$ and $\mathrm{Fe}$. Most lines have rest wavelengths of a few hundred Åbut some have wavelengths $\lambda \geq 900 \AA$, sufficient that they can be redshifted into the optical. The absorbing gas is normally transparent, except for Lyman continuum absorption, and dust extinction at the highest columns, so we can see hundreds of gas clouds along a line of sight. We see more absorption from higher redshifts, because the universe was denser then and the absorbing "clouds" have roughly constant proper size.

Understanding of absorption systems is advancing rapidly now, with observation of low redshift absorption by the Hubble Space Telescope, with high signal-to-noise spectra from the W.M. Keck observatory, and with better understanding of the Ly $\alpha$ forest from computer simulations.

\section{Types of Absorbers}

Absorption is seen from a wide variety of environments, from tenuous low density gas in the intergalactic medium and the very outer regions of galaxies, to the inner regions of galaxies and the immediate surroundings of QSOs. In Table 1 I give rough values for the neutral Hydrogen column densities $\mathrm{N}(\mathrm{H} \mathrm{I})$, the ionization, and the number of absorbers seen per unit redshift $N(\mathrm{z})$.

We are most likely to see absorption from gas which is very spatially extended. This gas usually also has a low density, so it is very sensitive to its environment. The sensitivity makes it extremely hard to predict the properties of the absorbing gas prior to observation, but instead we are learning to use observations to deduce the properties of the environment. 
TABLE 1. Types of Absorption System

\begin{tabular}{lllll}
\hline Origin & Name & $\begin{array}{l}\text { N(H I) } \\
\left(\mathrm{cm}^{-2}\right)\end{array}$ & $\begin{array}{l}\text { Ionization } \\
(\mathrm{H} \mathrm{II} / \mathrm{H} \mathrm{I})\end{array}$ & $N(\mathrm{z})$ \\
\hline IGM & Gunn-Peterson absorption & $<10^{12}$ & $>10^{6}$ & $>10^{3}$ \\
IGM & Ly $\alpha$ forest & $10^{12}-10^{14.5}$ & $10^{5}$ & $10^{3}$ \\
Outer galaxy halos & Ly $\alpha$ forest & $10^{14.5}-10^{17}$ & $10^{4}$ & $10^{2}$ \\
Inner galaxy halos & Lyman Limit Systems ${ }^{a}$ & $10^{17}-10^{20}$ & $10^{2}$ & 1 \\
Galaxy Disks & Damped Ly $\alpha$ & $10^{20}-10^{22}$ & $1-10$ & 0.1 \\
Near QSOs & BAL \& Associated & $<10^{17}$ & high & $1^{b}$ \\
\hline
\end{tabular}

${ }^{a}$ Including most metal lines systems with $\mathrm{C} \mathrm{IV}$ and $\mathrm{Mg}$ II absorption.

${ }^{b}$ Rate per QSO, not per unit $z$; about $10 \%$ of QSO spectra show broad absorption lines, and most have associated absorption which arises within Mpc of the QSO.

Consider the gravitational concentration of baryons into increasingly dense objects. In order of increasing gas density we have: the smooth intergalactic medium (the IGM is revealed in Gunn-Peterson absorption), filaments and foam in the IGM (seen as Ly $\alpha$ forest lines), the extreme outer parts of galaxies (Ly $\alpha$ absorption at $<160 h^{-1} \mathrm{kpc}$ ), the inner parts of galaxies (Lyman Limit Systems - LLS - and metal line absorption from $<30 h^{-1} \mathrm{kpc}$ ), inner parts of galaxies (damped Ly $\alpha$ absorption), and absorption near to QSOs (BAL - Broad Absorption Lines from gas ejected by QSOs, and associated absorption from QSO host galaxies or other near by galaxies). Since most gas is photoionized, as the density rises, the recombination time and level of ionization fall. Frequency of observation also falls with increasing $N(\mathrm{H} \mathrm{I})$, because denser gas clouds are smaller and rarer.

The BAL and associated absorption is different because it comes from a special environment. The intense radiation near to QSOs produces unusually high ionization, and the frequency of occurrence is a property of the QSOs, rather than the cosmological distribution of baryons.

\section{Connection to Galaxies: Baryon Flow}

As baryons flow from the smooth IGM into denser and denser objects there are three important feedbacks: radiation, heat and metals.

Ion ratios show that the absorbing gas is mostly photoionized by UV radiation. Much of this comes from QSOs and AGN and is "hard" with abundant far UV photons. Some near UV light also leaks out from young stars in galaxies, but the amount of this is unknown. Gas far from QSOs receives radiation which has a softer spectrum and reduced intensity because of absorption by intervening gas. The radiation determines the ionization 
of the gas, and the gas opacity shapes the spectrum and intensity of the radiation.

Star formation in galaxies heats and ejects gas, along with metals, into galaxy halos and presumably the IGM, where it is seen in absorption. We do not know whether most baryons are in galaxies and their halos, or in the IGM, since this depends in large part on the feedback of heat from star formation in galaxies. There will be more gas and metals in the IGM if the initial mass function favors high mass stars and many of these stars form together and over a short period of time in large bursts.

The radiation energy released by the stars which made the carbon seen in Ly $\alpha$ forest clouds with $\mathrm{N}(\mathrm{H} \mathrm{I})>10^{14.5}$ is also energetically significant (Madau \& Shull 1996), and might have helped re-ionize the IGM.

There is presumably dark matter associated with absorption systems, but we have not measured the ratio of baryonic to dark matter.

How sensitive are the properties of the absorbers to the parameters of galaxy formation: $\Omega_{b}$, the shape of the perturbation spectrum, the epoch and mechanism of IGM re-ionization, and the parameters of the background radiation field (origin, intensity, shape, spatial and temporal variation)? The answer should be obtained from detailed and accurate computer simulations of galaxy formation. In the long run we expect that observations of the absorbers (eg. rise in abundances and clustering with epoch, change in $\Omega$ in hot and cold gas) will constrain the free parameters of galaxy formation.

\section{Cosmological Distribution of Baryons and Metals}

In Table 2 I give a rough indication of the cosmological distribution of baryons and metals. The uncertainty in the level of ionization affects both the total baryon contents and the metal abundance, which is expressed in $\log$ solar units: $[\mathrm{M} / \mathrm{H}] \equiv \log (M / H)-\log (M / H)_{\text {solar }}$. The ionization depends on the gas density, and on the intensity and shape of the ionizing spectrum, which are not well known.

Entries in the table are from observations at different epochs. If the abundances in the gas seen in damped Ly $\alpha$ absorbers rises ten times from $z=2$ to today, and if galaxies eject gas so that the abundances in their halos and the IGM also rise ten times, then today the metals in the Ly $\alpha$ forest and IGM would be about 0.3 of those in galaxies, and perhaps most metals are outside the inner regions of galaxies. This is reasonable because $90 \%$ of the gas and metals in clusters of galaxies are in the inter-cluster medium. If galaxies eject metals on their own, e.g. in supernova driven winds, then most galaxies, both in and out of clusters, could eject most of their metals. 
TABLE 2. Cosmological Distribution of Metals

\begin{tabular}{lllll}
\hline Origin & $\log \Omega_{b}$ & {$[\mathrm{M} / \mathrm{H}]$} & $\log \Omega_{\text {metals }}{ }^{a}$ & $z$ \\
\hline IGM \& Ly $\alpha$ & $\leq-1.5$ & -2.5 & -6.0 & 2.8 \\
LLS & -3.0 & -2.0 & $-7.0^{b}$ & 3 \\
Damped Ly $\alpha$ & $-2.5^{c}$ & -1.0 & -5.5 & 2 \\
galaxies & -2.5 & 0 & -4.5 & 0 \\
\hline
\end{tabular}

${ }^{a} \log \Omega_{\text {metals }} \equiv \log \Omega_{b}+[M / H]-2$, all approximate values for $h=1$.

${ }^{b}$ For LLS both $\Omega_{b}$ and $[\mathrm{M} / \mathrm{H}]$ depend on the ionization. $\Omega_{\text {metals }}$ is better known than $\Omega_{b}$, or $[\mathrm{M} / \mathrm{H}]$, or the ionization. Data from Steidel (1990).

${ }^{c}$ The value given is twice that observed (Storrie-Lombardi \& Wolfe 1995), to correct for ionized hydrogen and systems missing because they contain dust which hides the background QSOs (Pei \& Fall 1995). Abundances from Pettini et al (1994).

\section{Missing Information}

The following are four areas where we expect important advances.

1. Measurement of the three dimensional shapes and sizes of absorbers; from observations of double and triple QSOs, UV spectra of spatially resolved galaxies, and the detection and mapping of $\mathrm{H} \alpha$ emission from LLS.

2. Determination of the ionizing spectrum; from the calibration of the proximity effect using faint QSOs and metal lines, and the measurement of the shape of the background spectrum using metal line ions.

3. Low $z$ identifications have great potential. Do all absorption systems with $\mathrm{N}(\mathrm{H} \mathrm{I})>10^{\mathbf{1 4 . 5}}$ arise in galaxies? Are metals always seen in absorption from the outer regions of galaxies? Are all damped systems disks? What types of galaxies produce each type of absorber? How is the gas moving relative to the galaxies?

4. Several "old" problems must be cleaned up: the effects of dust and lensing on damped Ly $\alpha$ absorbers at low $z$, the fraction of lines (e.g. narrow Ly $\alpha$ and metals) from ejected gas, measurement of temperatures from the comparison of the relative width of ions of different atomic mass, leading to assessment of collisional ionization, and the modeling of inhomogeneous absorbing regions.

\section{References}

Madau, P. \& Shull, J.M. 1996, ApJ, Feb 1

Pei, Y.C. \& Fall, S.M. 1995 ApJ

Pettini, M., King, D., Smith, L.J. \& Hunstead, R.W. 1994 in QSO Absorption Lines ed. G. Meylan, p.71

Steidel, C.C. 1990 ApJS, 74, 37-91

Verner, D.A., Barthel, P.D. \& Tytler, D. 1994, AApS, 108, 287 